\title{
Mechanical Circulatory Support Placement Timing
}

National Cancer Institute

\section{Source}

National Cancer Institute. Mechanical Circulatory Support Placement Timing. NCI

Thesaurus. Code C99539.

A description of when a mechanical circulatory support device was placed. 